\title{
Microbial Antagonism by Staphylococcus aureus
}

\author{
BY G. I. BARROW \\ Public Health Laboratory, Bradford
}

(Received 6 November 1962)

\begin{abstract}
SUMMARY
Seven strains antagonistic to indicator corynebacteria on solid media were found among 100 Staphylococcus aureus strains of human origin. These inhibitory strains all gave sharply defined inhibition zones, belonged to bacteriophage type 71, and were isolated from superficial infections. They were strongly active in direct antagonism tests against all the other 'aureus' strains, and against some coagulase-negative staphylococci, streptococci and other Gram-positive species; Gram-negative organisms were not susceptible. $S$. aureus strains producing hazy inhibition zones with corynebacteria showed similar though less extensive antibacterial activity. Inhibition by both kinds of staphylococci occurred under several different environmental conditions, but not in the presence of oleic acid. The antagonistic agents were relatively heat resistant, passed readily through cellophan, and showed considerable specificity in their action.
\end{abstract}

\section{INTRODUCTION}

It has long been known that some strains of staphylococci are capable of inhibiting the growth of other organisms, particularly corynebacteria, by the production of diffusible 'antibiotic' substances. The earlier literature was reviewed by Florey et al. (1949). Such strains were not thought to be associated with any particular kind of infection until Parker, Tomlinson \& Williams (1955) showed that most staphylococci of bacteriophage 'type 71', but very few others, were able to prevent the growth of Corynebacterium diphtheriae on solid media, with the formation of sharply defined zones of inhibition. These strains of staphylococci were closely associated with impetigo contagiosa, and were rarely isolated from deeper infections (Barrow, 1955; Parker et al. 1955). A number of different staphylococci with antagonistic properties formed wider inhibition zones with 'hazy' edges, but had little else in common. The few remaining strains giving sharp zones of inhibition possessed other ' 71 -like' characters, and were also associated with superficial skin infections (Parker, 1958). Later, Parker \& Simmons (1959) found that type 71 staphylococci were weakly active in deferred antagonism tests against other strains of Staphylococcus aureus. The present paper concerns further observations on the range and significance of inhibitory activity by type 71 staphylococci on solid media.

\section{METHODS}

\section{Organisms}

Staphylococcus aureus. One hundred strains of coagulase-positive staphylococci, isolated from routine bacteriological specimens, were phage-typed at the Public Health Laboratory, Leeds, by the method of Anderson \& Williams (1956). They 
were derived from infections such as boils, abscesses and skin lesions, as well as from ear, nose and throat swabs, and were representative of all the phage groups. In addition, two strains of Staphylococcus aureus antagonistic to corynebacteria were obtained from the National Collection of Type Cultures: NCTc 6507-strain 'Inhibitor' isolated from an empyema and deposited by A. Fleming in 1943; and NCTC 8004 -strain E 755, used by Gardner (1949). Each strain was examined for: (1) sensitivity to penicillin; (2) ability to inhibit the growth of corynebacteria on solid media; (3) production of opacity in horse serum agar medium, as described by Parker (1958); (4) production of opacity in egg-yolk broth (Oxoid), as described by Alder, Gillespie \& Herdan (1953). These strains were also used in various inhibition tests. They were kept on Lemco nutrient agar slopes and subcultured infrequently.

Coagulase-negative staphylococci. Twenty-five strains were used, of which fifteen were isolated from routine bacteriological specimens and nine from normal skin. The remaining culture, Staphylococcus saprophyticus (NCTC 7291), was originally isolated from healthy skin. Each strain was examined for inhibitory activity against, and susceptibility to, other organisms.

Corynebacteria. A suitable 'indicator' strain, highly susceptible to inhibition by type 71 staphylococci, was selected from a collection of corynebacteria obtained from routine specimens. This strain, an unnamed diphtheroid organism referred to as 'Bradford' (BFD) was isolated from an ear swab, and was a short broad rod, forming flat whitish colonies with lobate edges and a dull granular surface. It fermented glucose and sucrose only, and showed some enhancement of growth around zones of inhibition. It was thus similar to the original 'Bradford' organism sent to, and used by, Parker \& Simmons (1959). Later, a subculture of their nitratenegative avirulent strain of Corynebacterium diphtheriae mitis (no. 51 in the collection of the Public Health Laboratory, Manchester) was also obtained and used in parallel with the BFD diphtheroid. This 'Manchester' organism (MC) showed typical 'mitis' morphology and cultural characters. Both indicator organisms were subcultured weekly on blood agar medium and kept on the bench. Stock cultures were also kept at $4^{\circ}$.

Haemolytic streptococci. Twenty representative streptococci comprising different serotypes of group A and members of other Lancefield groups, originally isolated from an outbreak of throat infections and impetigo in a school (Barrow, 1961), were used. In addition 38 different strains of Streptococcus pyogenes, 20 of which possessed $M$ antigens, as well as 12 different strains from impetigo lesions, were obtained in the lyophilized state from the Streptococcus Reference Laboratory, Colindale. They were maintained by frequent subculture on blood agar medium, and were examined for inhibition of, and susceptibility to the action of, staphylococci.

Other organisms. These were used in various inhibition tests and included miscellaneous strains of pneumococci, viridans streptococci, enterobacteria and aerobic and anaerobic spore-bearing organisms isolated in the laboratory. One strain of Corynebacterium acnes (NCTC 737) was also used.

\section{Methods used in the study of inhibition on solid media}

Blood agar medium was used because it allowed good growth of Corynebacterium MC and because the zones of inhibition were easily seen. The blood also provided a source of catalase, thus excluding the possibility of inhibition due to peroxide 


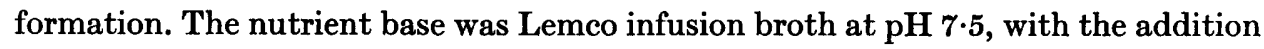
of $0.002 \%(w / v)$ cystine hydrochloride and $1.6 \%(w / v)$ New Zealand agar. Incubation was aerobic at $37^{\circ}$ overnight, unless otherwise stated.

\section{Direct antagonism}

In these methods, the active or test strain was placed on solid medium shortly after spreading a sensitive indicator organism over the surface. The term 'direct' is used instead of 'simultaneous' antagonism (Gratia, 1946) because it avoids the suggestion that each organism is necessarily active against the other.

(1) Stab inoculation. Small loopfuls of broth cultures of the test strains were deposited on the surface of media previously sown with indicator organisms. Later, the test strains were stab-inoculated with a straight wire throughout the depth of the medium. On incubation, the indicator organisms grew as confluent lawns, with zones of inhibition round some of the 'spot' or stab inocula. Stab-inoculation was simple, rapid and gave consistent results with wider inhibition zones than surface inocula. It was also eminently suitable for examining single colonies. In accordance with Parker \& Simmons (1959), inhibition of corynebacteria was considered positive $(\mathrm{DI}+)$ when the zones were $\mathbf{1 . 0} \mathrm{mm}$. or more in width; zones with sharply defined edges were designated $\mathrm{DI}+\mathrm{S}$, and those with hazy edges $\mathrm{DI}+\mathrm{H}$.

(2) Overlapping drop method. This was used to show competitive inhibition among staphylococci and was based on the cross-titration procedure of Rosebury, Gale \& Taylor (1954). Pairs of overlapping drops from dilutions of two different broth cultures were placed on solid medium with a standard loop. The second drop of each pair was added when the first had dried. The plates were examined after incubation for inhibition near the area of overlap.

\section{Deferred antagonism}

In these methods, the active or test organisms were grown on solid media before inoculation of the passive indicator strains. They allowed growth of the test and indicator organisms under different conditions, as well as permitting the detection of inhibitory substances sometimes not revealed by direct antagonism.

(1) Slide inoculation. Test strains were grown as central spots or as streaks across plates. After incubation, passive organisms were inoculated at right angles to the primary growth by means of glass microscope slides held horizontally between thumb and fingers. The long edges of sterile slides were seeded from culture-soaked filter papers in Petri dishes. This method allowed inoculation close to the primary culture without touching it.

(2) Colicine method. The technique of Abbott \& Shannon (1958) for typing Shigella sonnei by colicine production was used for staphylococci. After removal of the primary growth and exposure to chloroform, the passive organisms were quickly cross-inoculated by pressing glass slides alternately on culture-soaked filter papers as before and then on media. Later, a simple multiple-slide apparatus for the simultaneous and replicate inoculation of several different cross-streaks was developed for this and other purposes (Barrow \& Ellis, 1962). 
RESULTS

\section{Inhibition of corynebacteria}

The inhibitory activity of 100 strains of Staphylococcus aureus was first ascertained against the indicator corynebacteria by the 'spot' test. The results, with some properties of these staphylococci, are shown in Table 1. Seven strains were active and gave sharply defined zones of inhibition $(\mathrm{DI}+\mathrm{S})$ with both corynebacteria (Pl. 1, figs. 1, 2). These DI $+\mathrm{S}$ strains were isolated from superficial infections and comprised six type 71 cultures and one culture of phage pattern 55/71. They were re-tested at intervals, and the finding of Parker \& Simmons (1959) that DI +S cultures lost their inhibitory activity on storage and showed a simultaneous increase in susceptibility to group II bacteriophages, was confirmed. When dilutions of cultures showing loss of activity were sown on plates with the corynebacteria, some inhibitory staphylococcal colonies were detected among the many inactive colonies. These active colonies were indistinguishable from freshly isolated $\mathrm{DI}+\mathrm{S}$ cultures, and behaved in the same way on storage; active variants were never obtained from non-inhibitory colonies. The culture of phage pattern $55 / 71$ also yielded on dilution a mixture of DI $+S$ type 71 colonies and DI - colonies of pattern 55/71.

Table 1. Phage groups and some properties of 100 strains of Staphylococcus aureus isolated from routine specimens

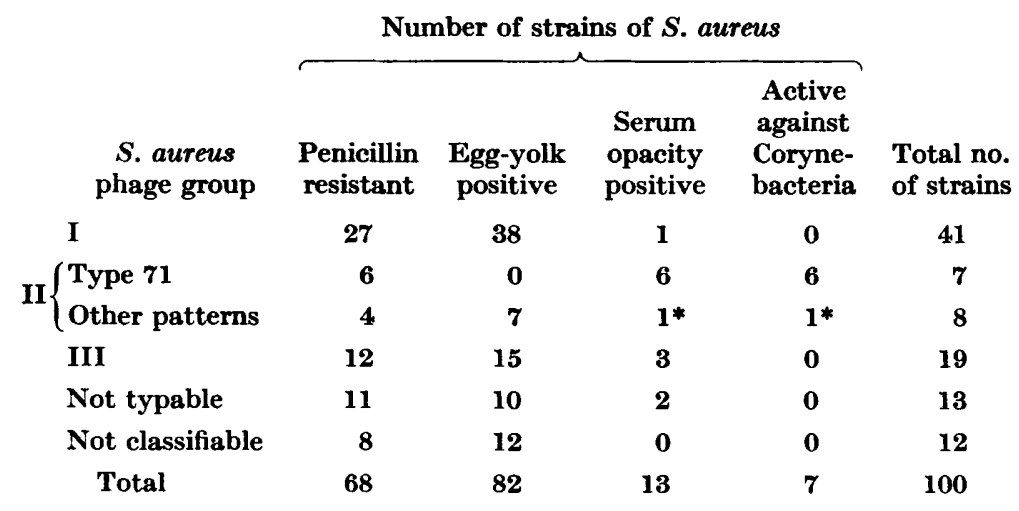

* This staphylococcal strain yielded inhibitory colonies of type 71 and non-inhibitory colonies of phage-pattern 55/71.

Because no inhibitory strains other than type 71 were identified, two 'aureus' strains active against corynebacteria were obtained from the National Collection of Type Cultures and used for comparison in some experiments. Strain NCTC 8004 (E 755), not susceptible to any of the typing phages in routine use, gave narrow inhibition zones with the BFD diphtheroid, and wide zones with hazy ill-defined margins (DI + H) with the MC diphtheria organism. Strain NCTC 6507 ('Inhibitor'), weakly susceptible to phage 29 , also gave wide hazy zones with organism Mc, but had little or no effect on the BFD organism (Pl. 1, figs. 1, 2). Neither of these aureus cultures showed much loss of inhibitory activity during storage. 


\section{(i) Staphylococcus aureus}

\section{Inhibition of staphylococci}

(a) Deferred antagonism. The active $(\mathrm{DI}+)$ type 71 strains of Staphylococcus aureus were first tested by the colicine method for inhibitory power against a few non-inhibitory (DI -) staphylococci. The DI $+\mathbf{S}$ type 71 strains inhibited the DI staphylococci. Next, these $D I+S$ strains and the two $D I+H$ NCTC strains were tested similarly against all the DI - staphylococci isolated from routine specimens, and then vice versa. In addition, 50 of the DI - strains were subsequently tested against each other. The combined results gave a chess board indicating mutual inhibitory activity. This showed that all the $D I+S$ type 71 strains inhibited the growth of every DI - strain, and conversely, no DI - strain had any inhibitory activity against $\mathrm{DI}+\mathrm{S}$ or $\mathrm{DI}+\mathrm{H}$ staphylococci. Each of the two $\mathrm{DI}+\mathrm{H}$ NCTC strains inhibited most, but not all, of the DI - staphylococci. Resistance to the

Table 2. Inhibitory activity between representative strains of Staphylococcus aureus in deferred antagonism tests

\begin{tabular}{|c|c|c|c|c|c|c|c|c|c|c|c|c|c|}
\hline \multirow{2}{*}{$\begin{array}{l}S . \text { aureus } \\
\text { phage } \\
\text { group }\end{array}$} & \multirow{2}{*}{$\begin{array}{l}\text { Primary } \\
\text { streak } \\
\text { S. aureus } \\
\text { strain no. }\end{array}$} & \multicolumn{12}{|c|}{ Passive cross-streaks $S$. aureus, strain no. } \\
\hline & & o. 1 & 2 & $\mathbf{3}$ & 4 & $\mathbf{5}$ & 6 & 7 & 8 & 9 & 10 & 11 & 12 \\
\hline $\mathbf{I}$ & $\begin{array}{c}1 \\
2 \\
(\mathrm{NCTC} \\
6507)\end{array}$ & + & - & $\bar{t}$ & - & + & $+\overline{+}+$ & $\overline{+}$ & - & $\bar{t}$ & - & - & - \\
\hline (Туре 71 & $\left\{\begin{array}{l}3 \\
4\end{array}\right.$ & $\begin{array}{l}++t \\
++t\end{array}$ & $\begin{array}{c}+++ \\
++\end{array}$ & - & - & $\begin{array}{l}++t \\
++t\end{array}$ & $\begin{array}{l}++t \\
++t\end{array}$ & $\begin{array}{l}+++ \\
+++\end{array}$ & $\begin{array}{l}+++ \\
+++\end{array}$ & $\begin{array}{l}+++ \\
+++\end{array}$ & $\begin{array}{l}++t \\
++t\end{array}$ & $\begin{array}{l}+++ \\
+++\end{array}$ & $\begin{array}{l}++t \\
++t\end{array}$ \\
\hline$\left\{\begin{array}{l}\text { Other } \\
\text { patterns }\end{array}\right.$ & Is $\left\{\begin{array}{l}5 \\
6\end{array}\right.$ & - & - & - & - & - & - & - & - & - & - & - & - \\
\hline III & $\begin{array}{l}7 \\
8\end{array}$ & - & - & - & - & - & - & - & - & - & - & - & - \\
\hline $\begin{array}{l}\text { Not } \\
\text { typable }\end{array}$ & $\begin{array}{c}9 \\
10 \\
(\text { NCTC } \\
8004)\end{array}$ & $\bar{t}$ & $\overline{-}$ & $\overline{ \pm}$ & $\overline{+}$ & $\overline{t+}$ & + & + & $\overline{+}$ & - & - & + & $\overline{+}$ \\
\hline $\begin{array}{l}\text { Not clas- } \\
\text { sifiable }\end{array}$ & $\begin{array}{r}11 \\
12\end{array}$ & - & - & $\overline{-}$ & - & - & - & - & - & - & - & - & - \\
\hline
\end{tabular}

$+++=$ Complete inhibition extending beyond area of overlap; $++=$ complete inhibition over area of overlap; $+=$ complete inhibition within area of overlap; $\pm=$ partial inhibition; $-=$ no inhibition.

action of the $\mathrm{DI}+\mathrm{H}$ strains was not, however, associated with any particular phage pattern. The inhibition zones produced by DI + staphylococci were distinct, although resistant colonies sometimes occurred within them. Among the DIstaphylococci only weak doubtful activity against occasional strains was observed. The results of a typical experiment with representative DI + and DI - organisms are shown in Table 2. When used as test organisms, active type 71 strains did not inhibit other $\mathrm{DI}+\mathrm{S}$ strains, nor did the $\mathrm{DI}+\mathrm{H}$ NCTC strains inhibit each other. Both DI $+\mathbf{H}$ strains, however, inhibited all the DI $+\mathbf{S}$ strains, and similarly each type 71 strain inhibited both $\mathrm{DI}+\mathrm{H}$ NCTC strains. Loss of inhibitory power by type 71 strains against corynebacteria coincided with loss of activity against 
staphylococci. Indeed DI - variants derived directly from DI +S type 71 strains were completely inhibited by the active parent cultures.

(b) Direct antagonism. The finding that DI $+\mathrm{S}$ type 71 staphylococci could inhibit the growth of all other strains of Staphylococcus aureus in deferred antagonism tests suggested that this ability might be of some advantage to them under natural conditions. It was important therefore to determine whether type 71 strains could suppress the growth of, or at least compete with, other staphylococci in direct antagonism experiments. With the spot test, inhibition was not generally observed when loopfuls of active type 71 broth cultures were deposited on lawns of DIstaphylococci. The effect of altering the ratio of organisms in the spot inocula and in the lawns was therefore investigated by the overlapping drop procedure. Undiluted DI + broth cultures readily inhibited diluted broth cultures of DI - strains, but not vice versa. When examined in this way, a few representative staphylococci, including the two NCTC strains, gave results corresponding with those of the deferred antagonism tests.

Although direct antagonism between staphylococci was thus demonstrated, this method was not satisfactory for examining numerous organisms, and a more suitable technique was therefore sought.

(c) Stab-inoculation method. It was observed that active type 71 staphylococci stab-inoculated with a straight wire throughout the depth of solid media inhibited the corynebacteria to a greater extent than similar surface spot inocula (Pl. 1, figs. 1, 2). When tested with lawns of staphylococci prepared from broth cultures, good inhibition of DI - by DI + strains was also observed, particularly when the straight wire was heavily charged with organisms from solid media. This technique was investigated and was found to provide a simple rapid method by which large inocula of one organism could easily be tested for direct antagonism against smaller inocula of other organisms.

It was thought that the smaller inhibition zones given by surface inocula were due to a decrease of the local antibiotic concentration by downward diffusion, and that antibiotic production throughout stab inocula gave higher surface gradients by diminishing this loss. This idea was supported by experiments in which antibiotic disks were placed on the surface, in the middle, and at the bottom of solid media, the surface of which had been seeded with a sensitive organism. After incubation, the surface disks gave narrower inhibition zones than the other disks. The importance of antibiotic loss by diffusion was emphasized by deferred antagonism experiments with media containing different concentrations of agar. Inhibition was weaker and the zones smaller in media containing $1 \%$ or less agar compared with media containing higher concentrations of agar. Diffusion was therefore limited by using $2 \%$ agar, by omitting the customary saline agar bases, and by making the depth of media slightly less than normal.

Using this technique, all the strains of Staphylococcus aureus were re-examined for direct antagonism against each other. The results were almost always the same as those obtained in deferred antagonism tests. All DI + staphylococci inhibited most of the DI - strains, including non-inhibitory variants derived from active type 71 cultures. Conversely, none of the DI - strains showed any inhibitory activity against other staphylococci. Again, both DI + H NCTC strains were susceptible to the $\mathrm{DI}+\mathrm{S}$ type 71 staphylococci; in turn these were inhibited, though to a lesser 
extent, by the Ncrc strains (Pl. 2, fig. 3). Neither Ncrc organism inhibited the other, nor were any of the DI $+S$ type 71 strains active against each other. By this method, type 71 strains were thus shown to be strongly and directly antagonistic to other 'aureus' strains.

\section{(ii) Staphylococcus albus}

Because of their remarkable inhibitory activity against other strains of Staphylococcus aureus, the interaction between type 71 staphylococci and 25 strains of coagulase-negative staphylococci was studied by the stab inoculation technique. Six of the $S$. albus strains were isolated from urines, four from urethral or vaginal discharges, and six from boils or other skin lesions. The remaining nine strains were from normal skin, one of which was $S$. saprophyticus NCTC 7291. It was found that 14 of the $S$. albus strains (including four from normal skin) were inhibited by type 71 stab inocula. On the other hand, only one of ten random DI - S. aureus strains was active in inhibiting three of the $S$. albus strains. Conversely, two coagulase-negative strains (both from normal skin) were active against all the type 71 strains, as well as against several DI - staphylococci. A few DI - S. aureus strains were also weakly susceptible to some (not necessarily the same) $S$. albus strains. When tested against the indicator corynebacteria, ten of the $S$. albus strains inhibited the BFD diphtheroid; two gave sharply defined zones of inhibition and eight gave hazy zones. Only three $S$. albus strains inhibited the Mc organism, all producing sharply defined zones; these included the two $S$. albus strains active against type 71 staphylococci. There was thus no close association as compared with $S$. aureus, between ability to inhibit corynebacteria and ability to inhibit other $S$. aureus strains. Loss of power to inhibit corynebacteria by type 71 strains coincided with loss of activity against coagulasenegative staphylococci, and resulted in susceptibility to other active $S$. albus strains.

\section{Inhibition of streptococci}

Interaction between type 71 staphylococci and different types of haemolytic streptococci was studied to try to assess their relative importance in impetigo. Twenty representative streptococci, including strains other than group $\mathbf{A}$, isolated from an outbreak of throat infections and impetigo in a school (Barrow, 1961) were examined for inhibition by type 71 stab inocula. Streptococci of group G were susceptible, but only doubtful activity was observed against occasional strains of Streptococcus pyogenes. Because $M$ antigens, necessary for complete type identification of Streptococcus pyogenes, were not found in most of these strains, other cultures with known $\mathbf{M}$ and/or $\mathbf{T}$ antigens, obtained from the Streptococcus Reference Laboratory, were investigated. These included 20 stock $M$ strains and $18 \mathrm{~T}$ strains of 'pyogenes', together representing 26 different serotypes. Also 12 different 'pyogenes' strains originally isolated from impetigo lesions and giving characteristic agglutination patterns, such as 3/13/B 3264, 8/IMP.19 and 5/11/27/44, were examined.

None of the 12 impetigo strains was inhibited by stab inocula of type 71 staphylococci on blood agar medium. Of the type strains of Streptococcus pyogenes, only the $M$ and $T$ cultures of type 23 were clearly and consistently inhibited by type 71 stab inocula. Most T strains were unaffected, although weak activity giving rise to hazy zones of minute colonies, was observed with several $\mathbf{M}$ strains. Examination 
of selected single colonies from streptococcal cultures revealed variation in susceptibility to type 71 staphylococci, but inhibition could not be related directly to colonial morphology nor to the presence of particular antigens. DI - staphylococci used as controls had little or no effect on any of the streptococci. Blood agar media at different $\mathrm{pH}$ values and incubated under different conditions did not give more definite results. Inhibition of every streptococcal strain, however, was produced on nutrient agar without blood by many staphylococci, including DI - cultures, and it was therefore not comparable with type 71 activity. Loss of ability by type 71 strains to inhibit corynebacteria resulted in loss of activity against $S$. pyogenes type 23, but weak activity against other streptococci was not affected. None of the streptococcal strains was active in deferred antagonism tests against type 71 staphylococci.

\section{Inhibition of other organisms}

Gram-negative species. Active staphylococci were tested by the stab-inoculation method against strains of Haemophilus, Neisseria, and several different enterobacteria. In no instance was inhibition observed.

Gram-positive species. Several organisms, including strains of pneumococci, enterococci and viridans streptococci, as well as different species of Corynebacterium and Bacillus were inhibited by type 71 staphylococci and by both NCTC DI $+\mathrm{H}$ strains. Under anaerobic conditions, different species of Clostridium were also inhibited by type 71 stab inocula, but not by the NCTC strains. One strain of Corynebacterium acnes (NCTC 737) was susceptible to the action of type 71 staphylococci, but not to the DI + H NCTC strains. Conversely, the 'acnes' culture was not active in deferred antagonism tests against these staphylococci.

\section{Properties of the inhibitory agents}

Resistance to heat. Plates with central streaks of type 71 staphylococcal growth were prepared for cross-inoculation by the colicine method, and were then placed in an anaerobic container. This was partly immersed in boiling water for $3 \mathrm{hr}$. The maximum air temperature reached inside the container was $83^{\circ}$. After removal, the plates were cooled and inoculated with sensitive indicator organisms. Inhibition occurred on incubation and was similar to that produced on unheated control plates, indicating that the type 71 inhibitory substance withstood this amount of heat. The two DI + H NCTC organisms also behaved in this way.

Diffusion through cellophan. Small sections of cellophan (Visking) tubing were autoclaved and then carefully dried. These sterile cellophan cylinders were placed upright in solid media just before setting. Active organisms were inoculated on the medium inside the cylinders, and indicator organisms outside-either at the same time as, or after incubation of, the active cultures. When plates were prepared in this way, growth of active staphylococci produced good inhibition of the Mc corynebacterium (Pl. 2, fig. 4), indicating that these inhibitory agents readily diffused through cellophan. This was also shown in direct and deferred antagonism experiments with sheets of commercial cellophan placed on the surface of solid media.

Specificity of action. All seven DI + S type 71 strains of staphylococci behaved in the same way and showed the same antibacterial range. With each, loss of activity against one organism always coincided with similar loss against other organisms, 


\section{Microbial antagonism by Staphylococci}

suggesting that their antagonistic activity was due to one and the same substance. This was, however, different from the inhibitory agents produced by the $\mathrm{DI}+\mathrm{H}$ NCTC organisms. Considerable specificity in their action was observed when DI+ strains were tested against colonies of staphylococci selected for their resistance to these inhibitory agents. Variants of DI - staphylococci resistant to one type 71 strain were equally resistant to the other type 71 strains, but they remained sensitive to the DI $+\mathrm{H}$ NCTC strains. Similarly DI - variants resistant to the DI $+H$ NCTC organisms were not resistant to type 71 strains. From type 71 organisms which had lost their inhibitory power, variants were selected which, unlike the parent culture, were resistant to active type 71 cultures, but which were not active against corynebacteria. These variants nevertheless remained susceptible to the $\mathbf{D I}+\mathbf{H}$ NCTC strains.

\section{Influence of environment on inhibitory activity}

A few experiments were made to determine whether type 71 staphylococci were active under some of the conditions which might be encountered in the skin. The factors considered were $\mathrm{E}_{h}, \mathrm{pH}$, temperature, salt concentration, the presence of unsaturated fatty acids, and the presence of blood, plasma or serum. Antibacterial activity by type 71 staphylococci was observed on solid media over a pH range from 5 to 9 after incubation at $37^{\circ}$ under aerobic and under anaerobic conditions. It also occurred and was almost as effective under these conditions when the organisms were grown at $25-30^{\circ}$, even in the presence of $5 \%(w / v) ~ N a C l$. In contrast, the $\mathrm{DI}+\mathrm{H}$ NCTC strains were not active when grown anaerobically. Inhibition by these staphylococci was detected by deferred antagonism methods after aerobic growth for as little as $5 \mathrm{hr}$.

Oleic acid was used to represent the unsaturated fatty acids present in sebum. Although not miscible with water, it was incorporated as fine globules in suspension by adding it to hot molten agar medium, mixing continuously until viscous, and then pouring rapidly on to plates. Some staphylococcal strains grew with difficulty on 0.5 or $1.0 \%$ oleic acid media, but type 71 strains, the DI $+\mathrm{H}$ NCTC organisms, and the BFD diphtheroid, grew well. Direct inhibition of corynebacteria or of DIstaphylococci was not observed on these plates. Inhibitory activity by $\mathrm{DI}+\mathrm{S}$ type 71 staphylococci in solid media was not affected by the presence of heated serum from several different animal species, or by human blood or pooled human plasma or serum. Inhibitory activity was not prevented by standard commercial staphylococcal antitoxin, or by an immune rabbit serum prepared against $D I+S$ type 71 organisms.

\section{DISCUSSION}

The present work confirms the finding of Parker (1958) that inhibition of corynebacteria by staphylococci, with the formation of sharply defined zones on solid media, is limited almost entirely to type 71 strains. These were all isolated from superficial infections, were penicillin resistant and produced opacity in horse serum agar but not in egg-yolk broth medium. An inverse relationship was found between the serum and egg-yolk opacity results, thus supporting Parker's conclusion that the serum opacity test provided a good indication of 'superficiality' among strains of Staphylococcus aureus. Gooder (1961) described a similar opacity reaction produced in broth by certain serotypes of Streptococcus pyogenes, including those 
associated with skin infections. The present work has also confirmed the findings of Parker \& Simmons (1959) that type 71 staphylococci lose their inhibitory activity on storage, and that this loss is accompanied by increased bacteriophage susceptibility. In contrast two staphylococcal strains which produced hazy zones of inhibition with corynebacteria remained stable.

Although Parker \& Simmons observed some activity by DI +S type 71 staphylococcal strains, but not by $\mathrm{DI}+\mathrm{H}$ strains, in deferred antagonism tests against other staphylococci they did not observe any inhibition by direct antagonism. They concluded that inhibitory activity between different staphylococci was weak and probably of little significance. However, it has been shown in the present work that with suitable methods type 71 staphylococci can be strongly and directly active against all other strains of Staphylococcus aureus, as well as against many other Gram-positive species. Two DI + H NCTC staphylococcal cultures were similarly active against many, though not all, strains of $S$. aureus. Stab inoculation was useful for detecting such activity; it allowed inhibition which would otherwise not have been observed and directed attention to the importance of limiting diffusion in order to maintain high local antibiotic concentrations. This is important when examining organisms with similar growth rates, since a critical antibiotic concentration may be attained before growth of the sensitive population reaches a resistant level, as was found by Cooper, Linton \& Sehgal (1958). These inhibitory agents were heatresistant, able to pass through cellophan, and showed considerable specificity in action when tested against resistant staphylococcal variants. Similar substances, probably polypeptides, were detected among staphylococci by Fredericq (1946) and by Halbert, Swick \& Sonn (1953). Many of these differed only in heat stability or in specificity of action, and were thus analogous to the colicines produced by enterobacteria (Fredericq, 1957). The similarity in behaviour of each type 71 strain, and the fact that loss of power against one organism coincided with similar loss against other organisms suggested that one particular substance was responsible for their activity. Type 71 staphylococci were active under most of the conditions likely to be encountered in the skin, and were also susceptible to the action of some commensal organisms. Nevertheless, as with other infections, there is still no proof that interaction between invading and resident organisms is actually concerned in the initiation of lesions.

Staphylococci isolated from human secretions and active against mucoid colonies of Streptococcus pyogenes were described by Murray \& Loeb (1950). In the present work, little evidence was obtained with laboratory strains of 'pyogenes' to support the hypothesis that type 71 staphylococci suppress the growth of some streptococcal serotypes, thus giving an apparent association of other streptococci with impetigo. The streptococcal strains associated with superficial infections may, however, be better able to survive in the skin. Although microbial antagonism could not be related directly to infection, it is possible that, once established, type 71 staphylococci may prevent subsequent invasion of impetigo lesions by other organisms.

I am grateful to Dr H. G. Smith for advice and encouragement during this work; to Dr G. B. Ludlam, Public Health Laboratory, Leeds, for phage-typing staphylococci; and to Mr P. Harrison, Medical Photography Department, Bradford Royal Infirmary for the photographs. 


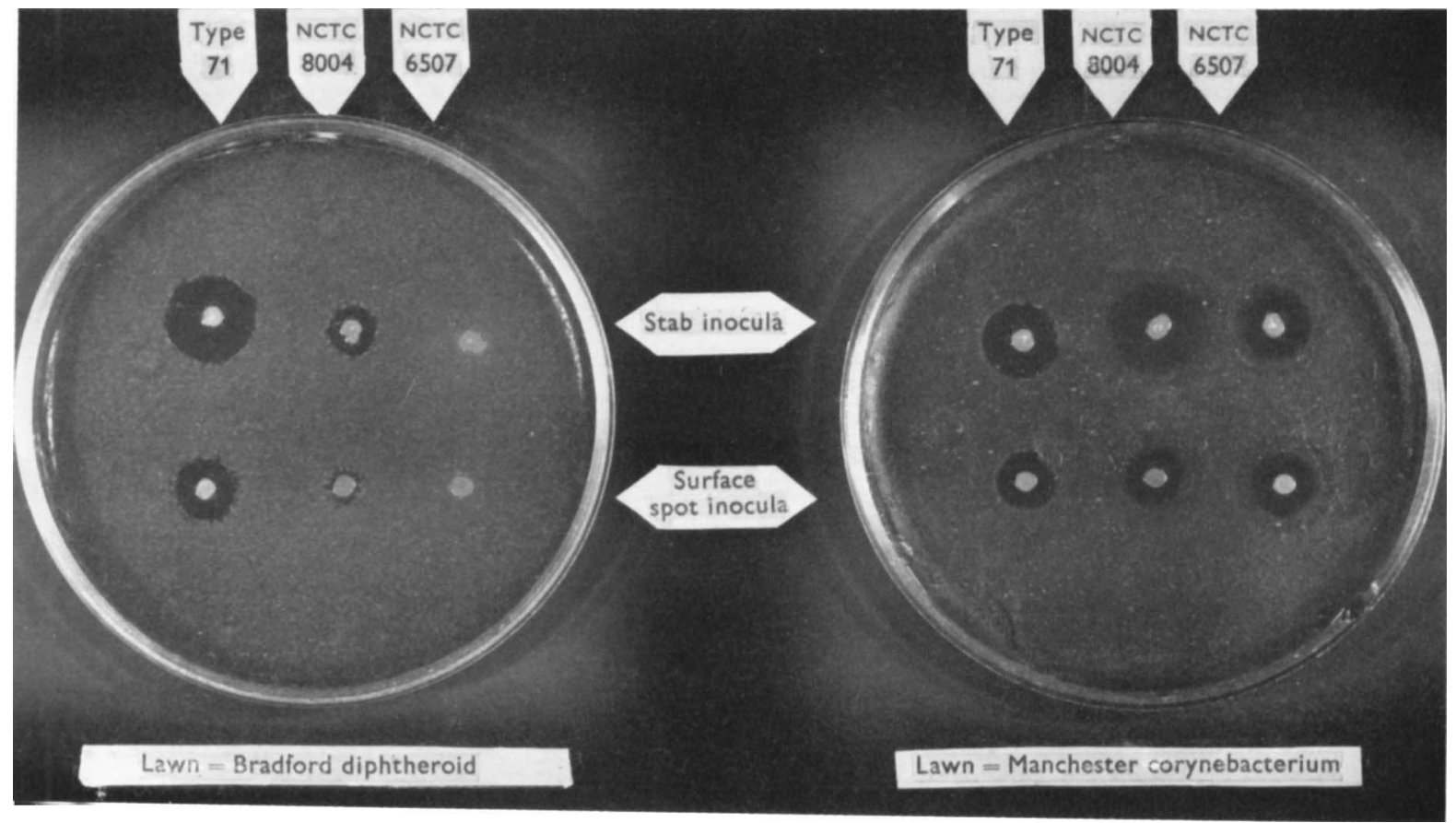

Fig. 1 


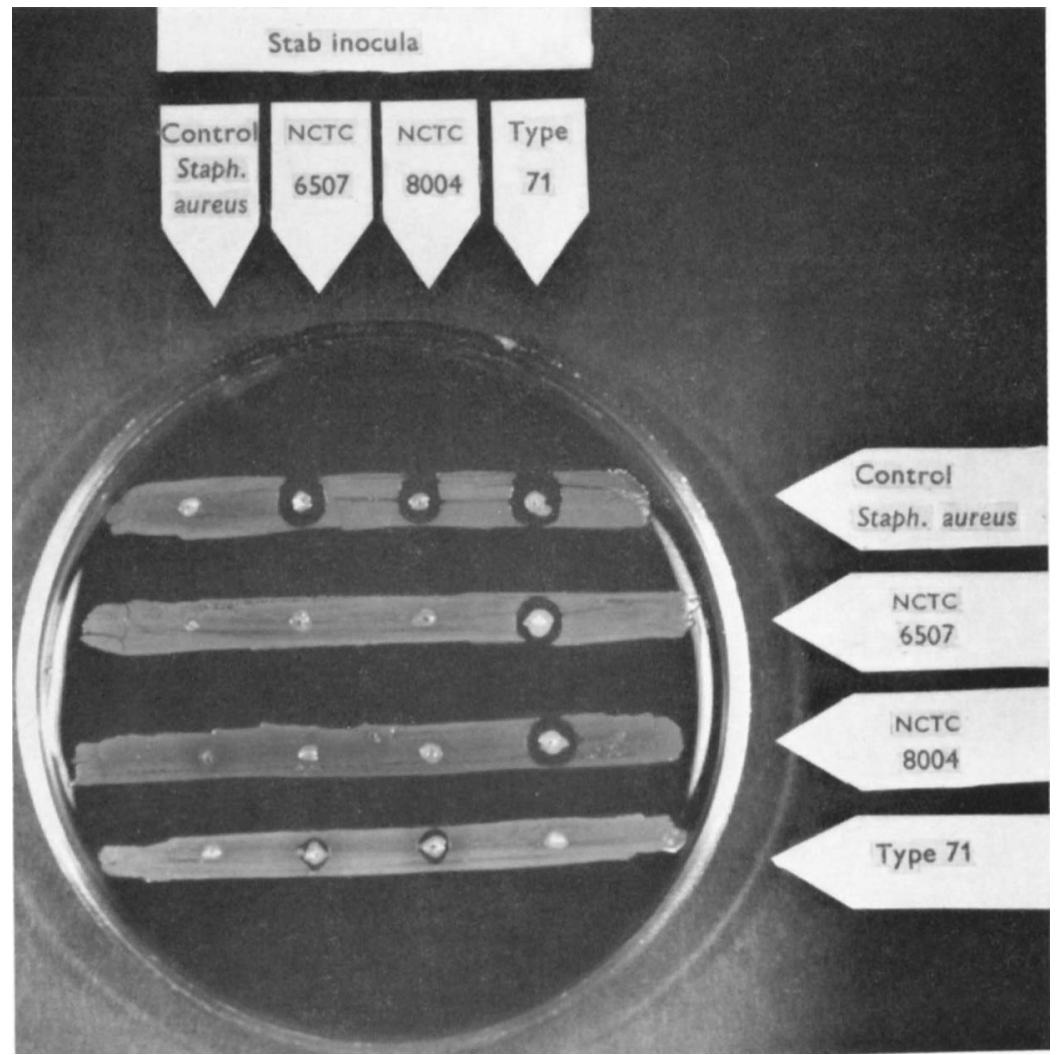

Fig. 2

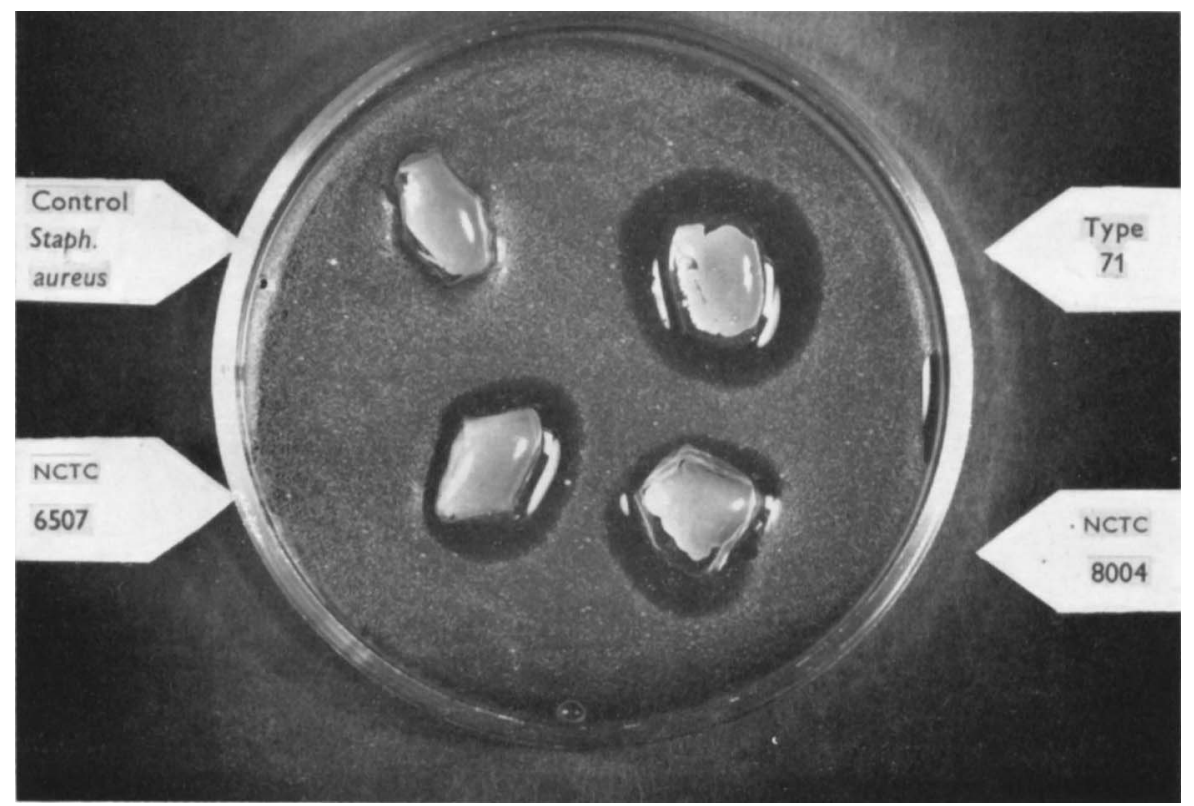

G. I. BARROW

Fig. 3 


\section{REFERENCES}

Aвbott, J. D. \& Shannon, R. (1958). A method for typing Shigella sonnei using colicine production as a marker. J. clin. Path. 11, 71.

Alder, V. G., Giluespie, W. A. \& Herdan, G. (1953). Production of opacity in egg-yolk broth by staphylococci from various sources. J. Path. Bact. 66, 205.

Anderson, E. S. \& Williams, R. E. O. (1956). Bacteriophage typing of enteric pathogens and staphylococci, and its use in epidemiology. A review. J. clin. Path. 9, 94.

Barrow, G. I. (1955). Clinical and bacteriological aspects of impetigo contagiosa. J. Hyg., Camb. 53, 495.

BARrow, G. I. (1961). An outbreak of streptococcal infection caused by different strains. Mon. Bull. Minist. Hlth Lab. Serv. 20, 224.

Barrow, G. I. \& Eusus, C. (1962). Colicine typing of Shigella sonnei by replicate multipleslide inoculation of indicator organisms. Mon. Bull. Minist. Hlth Lab. Serv. 21, 141.

Cooper, K. E., Linton, A. H. \& Sehgal, S. N. (1958). The effect of inoculum size on inhibition zones in agar media using staphylococci and streptomycin. J. gen. Microbiol. 18, 670 .

Florey, H. W., Chain, E., Heatley, N. G., Jennings, M. A., Sanders, A. G., Abraham, E. P. \& Florey, M. E. (1949). Antibiotics, 1, 493. London: Oxford University Press.

FrédéricQ, P. (1946). Sur la sensibilité et l'activité antibiotique des staphylocoques. C.R. Soc. Biol., Paris, 140, 1167.

FrédéricQ, P. (1957). Colicins. Annu. Rev. Microbiol. 11, 7.

GARDNER, J. F. (1949). An antibiotic produced by Staphylococcus aureus. Brit. J. exp. Path. 30, 130.

Gooder, H. (1961). Association of a serum opacity reaction with serological type in Streptococcus pyogenes. J. gen. Microbiol. 25, 347.

Gratia, A. (1946). Techniques sélectives pour la recherche systématique des germes antibiotiques. C.R. Soc. Biol. Paris, 140, 1053.

Halbert, S. P., Swick, L. \& Sonn, C. (1953). Characteristics of antibiotic-producing strains of the ocular bacterial flora. J. Immunol. 70, 400.

MurRay, R. G. E. \& Loes, L. J. (1950). Antibiotics produced by micrococei and streptococci that show selective inhibition within the genus Streptococcus. Canad. J. Res. (E), 28, 177 .

PARker, M. T. (1958). Some cultural characteristics of Staphylococcus aureus strains from superficial skin infections. J. Hyg., Camb. 56, 238.

Parker, M. T. \& Simmons, L. E. (1959). The inhibition of Corynebacterium diphtheriae and of other Gram-positive organisms by Staphylococcus aureus. J. gen. Microbiol. 21, 457.

Parker, M. T., Tomlinson, A. J. H. \& Williams R. E. O. (1955). Impetigo contagiosa. The association of certain types of Staphylococcus aureus and of Streptococcus pyogenes with superficial skin infections. J. Hyg., Camb. 53, 458.

Rosebury, T., GaLe, D. \& TAYlor, D. F. (1954). An approach to the study of interactive phenomena among micro-organisms indigenous to man. J. Bact. 67, 135.

\section{EXPLANATION OF PLATES}

Plate 1

Figs. 1, 2. Results given by three active strains of Staphylococcus aureus tested for direct antagonism against indicator corynebacteria. With the Manchester organism, type 71 strains gave sharply defined inhibition zones $(D I+S)$, whereas the two NCTC strains gave zones with hazy ill-defined margins (DI $+\mathbf{H})$. Stab inocula produced wider zones than similar surface spot inocula.

\section{Plate 2}

Fig. 3. Results given by stab inocula of one control (DI-) and three active strains of $S$. aureus tested against each other for direct inhibition. The parallel streaks represent growth from single loopfuls of broth cultures. The stab inocula were from growth on solid media.

Fig. 4. Direct inhibition of the Manchester corynebacterium by diffusion of antibiotics produced by three strains of $S$. aureus through cellophan tubing cylinders placed in the medium before setting. 